\title{
Invasive Aspergillosis Following Liver Transplantation and Its Successful Management
}

\author{
Authors \\ S R Karthick ${ }^{1}$, Kanimozhi Rathinasamy ${ }^{1}$, Satish Logidasan ${ }^{2}$, Arulraj Panchatcharam ${ }^{3}$ \\ Gowrishankar Anjaneyan ${ }^{4}$, Karthik Prakasam ${ }^{5}$, PrasanaVadhanan ${ }^{6}$, Ambal S $^{7}$ \\ 1,2,3,4,5,6,7 Department of Anesthesiology, Govt Stanley Medical College, TN- 600001, India \\ Corresponding Author \\ Satish Logidasan \\ Department of Anesthesiology, Govt Stanley Medical College, TN- 600001, India \\ Email: drsatishlogi@gmail.com
}

\begin{abstract}
Oppurtunistic infections represent a major cause of mortality and morbidity following liver transplantation. Mortality rate is high for invasive fungal infections following liver transplantation, candidiasis is the most common among them, followed by aspergillosis. Incidence of Invasive aspergillosisis 1 to $8 \%$ in liver transplant recepients. The major trap in the scenario is that these infections are difficult to diagnose as the symptoms and signs are highly non specific.

Here we present how to diagnose and manage invasive aspergillosis involving the abdomen in a diabetic patient after deceased donor liver transplantation

Keywords: Invasive aspergillosis, Liver transplant, Fungal infections.
\end{abstract}

\section{Introduction}

A 57 year old male patient came to the liver transplant outpatient department with a cryptogenic DCLD. He came under Child Pugh C status. His MELD (Model for End Stage Liver Disease) score was 21 . He belonged to $\mathrm{O}+$ ve blood group. Liver transplantation was advice for him as final treatment. DDLT was done for a patient aged 57, with MELD 21. He was a diagnosed case of Type II Diabetes mellitus for the past 10 years under control with oral hypoglycaemic agents. He was an alcoholic and smoker for the past 20 years.

After getting a suitable deceased donor of the $\mathrm{O}+\mathrm{ve}$ blood group, he was planned for liver transplantation. Consent was obtained from the patient and attenders and he was wheeled inside the operating room. Total duration of surgery was 12 hours. Blood products were replaced based on periodic TEG monitoring. Blood loss was $2000 \mathrm{ml}$ during the entire course of surgery. Totally 6 PRBCs, 9 FFPs, 3 cryoprecipitate and around 350 $\mathrm{ml}$ of SDP (single donor platelets) were transfused for him. Reperfusion was uneventful.

\section{Postoperative Course}

Patient was shifted to postoperative Liver transplant ICU after completion of procedure. $\mathrm{He}$ was hemodynamically stable with minimal noradrenaline support. In the immediate postoperative period his $\mathrm{Hb}$ was 9 gms\% and platelets were 82,000 cell 
per cu. mm. CRP was 79.1 on the same day. He was started on injection meropenom, teicoplanin and fluconazole intravenously. Injection Albumin 20\% infusion was on flow.

On the first postoperative day he was gradually weaned from mechanical ventilation and extubated at $11 \mathrm{am}$ in the morning. There was a fall in lactate from 4.6 to 2.6 in the first postoperative day. USG Doppler of the abdomen showed a normal study with a good PV flow except for a minimal pleural effusion. Day 1 he was started on tacrolimus at the dose of $1 \mathrm{mg}$ twice daily.

On the third postoperative day, USG Doppler showed a left subhepatic collection of around 300 $\mathrm{ml}$. His abdominal girth was $90 \mathrm{~cm}$. The next day abdominal girth again raised to $92 \mathrm{~cm}$.

There was a steady rise in platelets hence he was started on additional immunosuppresion with oral mycophenolate mofetil 500mg twice daily along with once daily oral prednisolone $20 \mathrm{mg}$. The patient was clinically stable except for a further raise in abdominal girth. From the fifth postoperative day there was a rising trend of sugars for which insulin infusion was started. Meanwhile the tac levels were lesser requiring a bit of higher immunosuppresion.

On the $10^{\text {th }}$ postoperative day the tac levels were only 3.6 and hence tablet tacrolimus dose was raised to $2.5 \mathrm{mg}$ twice daily. On the same day patient developed a temperature of 103 degrees F. There was minimal pus discharge mixed with serous content from the wound. Biopsy was sent from the wound.

\section{Relaporotomy for Invasive Aspergillosis}

On the $11^{\text {th }}$ postoperative day fever persisted for the patient. Wound biopsy report came as invasive fungal infection. CECT Abdomen / Chest / Brain were taken immediately to rule out aspergillosis at other sites. All the three were normal. On the same day patient was taken for relaparotomy under general anaesthesia.

\section{Intra operative findings were}

1. Yellowish white lesion noted in lower flap of horizontal part of incision $2 \times 1.5 \mathrm{~cm}$ invading part of omentum $4 \times 4 \mathrm{~cm}$ and serosal layer of hepatic flexure $1.5 \times 1 \mathrm{~cm}$.

2. Around $300 \mathrm{ml}$ coagulum found anterior to left lobe of liver

3. Graft was normal

4. Free fluid of around $500 \mathrm{ml}$ was drained

Entire horizontal part of the wound was excised with1 $\mathrm{cm}$ of safe margin, except at one point where > $2 \mathrm{~cm}$ clearance was given. Partial omentectomy was done at involved site. Full thickness sleeve colectomy done at hepatic flexure giving a marigin of $0.5 \mathrm{~cm}$ and closed in 2 layers. Amphotericin - B poured in peritoneal cavity. Vertical part of the wound was closed and stay sutures applied applied for horizontal portion. Injection Amphotericin - B was added at a dose of $250 \mathrm{mg}$ IV once daily and patient shifted to post operative LICU. He was extubated on the same day.

\section{Antifungal Therapy}

Next day the patient was started on oral feeds. He was planned to maintain low TAC levels to prevent further flaring up of fungal infections. The liver pathologist gave the tissue biopsy result as invasive fungal infection. Inj. Ambisome was continued. It was discussed with Infectious disease consultant, we learnt that tissue staining cannot help identifying fungal species which is essential for initiating treatment. So tissue culture was sent to the microbiology department. Injection voriconazole was added,300 mg twice daily followed by $200 \mathrm{mg}$ twice daily intravenously second day onwards. Culture confirmed Aspergillus species. Patient was put on tight glycemic control. Barrier nursing was followed. Strict antimicrobial prophylaxis carried out. Hand hygiene practices were strengthened for the attending physicians. Entry to the LICU were restricted. Wound dressing was done by the same surgeon twice daily for the next 10 days. Tacrolimus dosage was tailored based on once in 3 days TAC assay values. The requirements of tacrolimus were also lesser day by day. Amphotericin was continued till 14 th day and then stopped. 10 days after the relaparotomy the patient had loss of appetite and was lethargic. Differential 
count taken that day revealed eosinophilia where in suspicion of rejection came into play. Liver biopsy was taken and proved rejection.

As the patient was recovering from a fungal insult, pulse steroid therapy was avoided and immunosuppresion was escalated. Mycofenolate mofetil (MMF) was raised to $500 \mathrm{mg}$ twice daily. After 22 days of intravenous voriconazole, it was changed to tablet voriconazole $200 \mathrm{mg}$ twice daily and was advised to continue for 3 to 6 months. Secondary suturing of the skin completed 25 days after relaparotomy. Later patient was discharged with oral voriconazole, immunosuppresants, nutritional supplements, aspirin for thrombo prophylaxis. He is under close follow up till now.

\section{Discussion}

Liver transplantation is the accepted therapy for any form of fulminant liver failure. Earlier in 1960s and 1970 s attempts of liver transplantation resulted in poor survival[ 1 year survival rate $30-35 \%]^{1}$. Currently with the advances in monitoring and immunosuppresion, liver transplantation has a 1, 3 and 5 year survival rates of $88 \%, 80 \%$ and $75 \%$ respectively ${ }^{2}$. The major chunk of this success is favoured by reduction in allograft rejection, which inturn is done by tailored immunosuppresion. Despite measures like antimicrobial prophylaxis, barrier nursing, vaccination infections still represent a major cause of morbidity and mortality post liver transplantation $^{3}$. It is estimated that upto $80 \%$ of liver recepients will develop at least one infection during the first year post liver transplantation. Most of these infections are successfully treated, but some infections are grave ${ }^{4}$.

Amongst these infections fungal infections are relatively common and more dangerous. The major complicating factor is delayed diagnosis of fungal infections. The symptoms are non specific and even patients with disseminated disease with multiple organ involvement might not present with organ specific symptoms. Among the fungal infections, candida tops the list. Aspergillus species are the second most common fungal pathogen responsible for infection in liver recepients5. The mortality rates for liver recepients with invasive aspergillosis exceeds $90 \%$.

Aspergillus species accounts for one quarter of invasive fungal infections. They are airborne in all environments, both inside and outside the hospitals. The inhalation of spores through airborne route results in pulmonary infections with extrapulmonary dissemination to various other organs. Only a few species cause illness in humans. They are A. 30836fumigatus (most common), A. niger, A. flavus and A. fureus. For aspergillus infection to occur the patients immunological status plays a pivotal role ${ }^{7}$. Our patient being a liver transplant recipient under cover of immunosuppresion in addition a type II Diabetic has been a suitable host for aspergillus species to invade. The diagnosis of aspergillosis can be elusive because the fungus is very difficult to isolate and systems and signs are very non specific. Most infections occur during $1^{\text {st }}$ year of transplantation. The median time to diagnose is 100 days ${ }^{8}$.

In a a study of 152 transplant recepients, Briege et al identified two independent risk factors for systemic fungal infections. They are the amount of FFP transfused due to poor initial allograft function and acute renal failure requiring hemofiltration and hemodialysis ${ }^{9}$. Other risk factors being fuminant hepatic failure, CMV disease and prolonged ICU stay.

After suspecting the possibility of invasive aspergillosis based on the above risk factors and clinical findings, should be confirmed by one of the following.

(1) LRTI symptoms with risk factors, CT image showing well circumscribed with or without a halo sign, air crescent sign or cavity (2)CNS infections with focal lesions.(3)Recovery by culture of the mould. Sensitivity of fungal cultures is relatively low, hence detection of aspergillus antigens like galactomannan in clinical samples like plasma, serum, bronchoalveolar lavage fluid or CSF is a useful diagnostic tool. Whereas there is a risk of false positivity if the patient is under cover of Beta lactam antibiotics. 
The EORTC - MSG criteria decifies the probability and possibility of invasive fungal disease.

Probable IFD (Invasive fungal disease) requires presence of a host factor, a clinical criterion and a mycological criteria.

Possible IFD includes all, but lacks a mycological criteria. Our patient fulfilled the probable criteria as mycological isolation was possible. The patient doesn't satisfy the clinical criteria as CT chest/CT abdomen/ CT brain were normal. Whereas the strong suspicion of invasive fungal infections must be kept in mind are uncontrolled Diabetes mellitus, Immunosuppresion, Long term steroid therapy, Fungal isolation from scrapings

Though the patient was receiving fluconazole as a routine antifungal prophylaxis as every other post operative liver transplant patient, generally most of the aspergillus species are resistant to fluconazole. Amphotericin - B is usually used to treat fatal fungal infections. Amphotericin - B has been used conventionally for treating invasive aspergillosis. A large randomized comparative study was done by Herbrecht et al to study the efficacy of 2 different drugs in primary treatment of invasive aspergillosis. The two drugs were Amphotericin B and a broad spectrum antifungal voriconazole. The study concluded the superiority of voriconazole over Amphotericin - B in terms of response rate, survival rate and safety. Hence voriconazole was chosen for our patient though Amphotericin - B was poured in peritoneal cavity on reopening.

Antifungal prophylaxis generally remains a controversial issues in post solid organ transplantation patients. Fluconazole and echinocandins are both used for targeted prophylaxis. Echinocandins have become the leading choice as they have fungicidal activity and they have no interaction with calcineurin inhibitors ( ). The use of antifungal prophylaxis in high risk patients has reduced the incidence of fungal infections, but has not led to improvement in overall mortality in many series ( ). In our institution we use fluconazole as a routine prophylaxis.

\section{Conclusion}

To conclude in a post transplant recipient, invasive aspergillosis is invariably fatal. Apart from stringent infection control measures, tight glycemic control, anti fungal prophylaxis, early element of suspicion, tailored immunosuppresion and aggressive treatment are manadatory in a case of invasive aspergillosis following liver transplantation. 